\title{
El plagio: consideraciones para su prevención
}

\author{
Elizarda Vargas-Morúa * \\ https://orcid.org/0000-0002-2255-0340
}

Recibido: 20 de agosto de 2020 • Aceptado: 4 de febrero de 2021

\section{Resumen}

Este trabajo corresponde a un estudio bibliográfico, descriptivo y exploratorio para analizar el concepto de plagio, así como los tipos y factores que inciden en su práctica. Se enfatiza en la importancia de la prevención mediante la enumeración de prácticas viables identificadas por personas investigadoras para su aplicación en centros educativos. Adicionalmente, se hace una breve mención a la detención y sanción como conceptos.

Asimismo, se incorpora una resolución de la Sala Constitucional de Costa Rica promulgada en el 2020, en la cual, con base en el principio de la Autonomía Universitaria, se da respaldo a las universidades para la realización de pruebas virtuales asincrónicas y sincrónicas, incluso con grabación de imagen y voz, bajo ciertas condiciones, en relación con el caso en concreto.

Con este trabajo se espera contribuir con la presentación de alternativas viables para prevenir el plagio, así como inquietar respecto del trabajo que pueden desarrollar las instituciones educativas para instruir y concientizar al alumnado respecto de la importancia del conocimiento, la demostración y comprensión de lo aprendido para un desarrollo profesional pleno.

Palabras clave: Fraude académico, honestidad académica, sanciones disciplinarias.

* Licenciada en Derecho de la Universidad de Costa Rica, de Costa Rica. Máster en Administración de Empresas de la Universidad Latinoamericana de Ciencia y Tecnología, de Costa Rica. Profesora en la Escuela de Ciencias de la Administración de la Universidad Estatal a Distancia (UNED), de Costa Rica. Es asesora en la Vicerrectoría Académica de la UNED y desde el 2019 integra la Comisión de Carrera Profesional de la UNED. Correo: evargasm@uned.ac.cr 


\title{
Plagiarism: Preventive Guidelines
}

\begin{abstract}
This text is a bibliographic, descriptive and exploratory study used to analyze the concept of plagiarism, types of plagiarism, as well as factors that come into play in the act of plagiarizing. The emphasis is put on the importance of preventing plagiarism through listing viable practices which are identified by researchers and putting them into practice in educational establishments. In addition to that, a brief mention of the concepts of detention and penalty is given.

Moreover, a motion enacted in 2020 from the Constitutional Chamber of Costa Rica is incorporated, in which, based on the principle of University Autonomy, support is given to the universities to carry out virtual quizzes both synchronously and asynchronously, even with voice and image recording, under certain conditions, and in relation to this particular case.

With respect to this text, the hope is to contribute to the presentation of viable alternatives in the prevention of plagiarism. It is also the hope to raise concern regarding the work that educational establishments should develop to instruct and create consciousness among the student body in regards to the importance of knowledge, and the demonstration and comprehension of what is learned for complete professional development.
\end{abstract}

Key words: Academic fraud, academic honesty, disciplinary sanctions.

\section{Le plagiat: quelques considérations pour le prévenir}

\section{Résumé}

Ce travail correspond à une étude bibliographique, descriptive et exploratoire qui analyse le concept de plagiat, ainsi que les facteurs qui incident dans sa pratique. Il fait le point sur l'importance de le prévenir moyennant l'énumération des pratiques viables et identifiées pour personnes chercheuses en vue de sa mise en place dans les institutions éducatives. D'ailleurs, il fait une courte mention sur la détention et la sanction en tant que concepts.

Également, le travail comprend la résolution de la Chambre Constitutionnelle du Costa Rica, promulguée en 2020, dans laquelle, sur la base de l'autonomie universitaire, les universités peuvent s'y appuyer pour réaliser des épreuves virtuelles. Ces épreuves peuvent être synchrones et asynchrones, même avec enregistrement d'image et de voix, selon certaines conditions, tout en rapport avec ce cas qui a ouvert la voie légale.

Finalement, cet article a pour but de proposer des alternatives viables afin de prévenir le plagiat, de sensibiliser les institutions éducatives sur le travail qu'elles peuvent mener pour instruire et éveiller les consciences des étudiants concernant l'importance des connaissances, la démonstration et la compréhension de ce qui a été appris à l'effet d'un plein développement professionnel.

Mots-clés: Fraude académique, honnêteté, sanctions disciplinaires. 


\section{Introducción}

Con la llegada de la pandemia en el 2020 y con la virtualización de las actividades académicas, el tema de las faltas disciplinarias por fraudes académicos en las pruebas ha cobrado importancia, debido a la reincidencia de este tipo de vicios, principalmente con el plagio, en las asignaciones académicas, así como la copia en los exámenes en línea.

La forma masiva en que estos problemas disciplinarios se realizan hoy y la afectación que conlleva a las diversas actividades académicas, convoca nuevamente a la reflexión respecto a cómo deben actuar los docentes, los factores que inciden en estas prácticas, cómo prevenir y controlar eficazmente el problema, o qué tipo de actividades académicas orientativas se pueden realizar para evitar que los estudiantes incurran en tales faltas disciplinarias.

La realidad de nuestros tiempos, con las facilidades tecnológicas, aunada a la presión económica y de estudio, ha hecho que se evidencie cada vez más el uso de recursos impropios para la elaboración de las actividades académicas. Con este estudio bibliográfico, relativo a investigaciones sobre el plagio, se pretende conocer los recursos que los investigadores han identificado para prevenir el plagio entre los estudiantes y conocer las propuestas realizadas a nivel de prevención, principalmente. Adicionalmente, se hace breve mención a la detención y sanción.

Con el presente texto se pretende además coadyuvar al trabajo que se realiza desde la Vicerrectoría Académica de la UNED y la Defensoría de los Estudiantes en la prevención del problema, así como del esfuerzo individual que realizan muchos profesionales de la educación.

\section{Desarrollo}

Cada institución educativa puede regular lo que se conoce como faltas disciplinarias. Un vicio muy común a lo interno de las comunidades estudiantiles es el plagio. Este puede estar presente en instrumentos de evaluación tales como tareas, trabajos de investigación y pruebas escritas.

Desde hace tiempo, se viene reconociendo que este es un problema recurrente en la educación superior ${ }^{1}$. De ahí la importancia de conocer el problema y proporcionar instrucciones claras a los estudiantes sobre la responsabilidad que conlleva la escritura de trabajos académicos.

\footnotetext{
${ }^{1}$ Bob Price, «Avoiding plagiarism: guidance for nursing students», Europe PMC 28, n. ${ }^{\circ} 26$ (2013): 4551. doi:10.7748/ns2014.02.28.26.45.e8514
} 


\section{Delimitando el plagio}

\section{Concepción del plagio}

La palabra plagio, tiene diferentes acepciones, una de ellas es: «Copiar en lo sustancial obras ajenas, dándolas como propias $»^{2}$. Algunos autores ${ }^{3}$ distinguen entre el plagio por negligencia (no citar o referenciar correctamente) y el plagio por deshonestidad (copiar en un examen). Sin embargo, y como se verá adelante, para efectos de la normativa, ambos son igualmente reprochables y sancionables.

En la literatura, se ha definido el plagio de diversas formas:

1. Reutilizar sin atribución.

2. Uso injusto del conocimiento, ya sea intencionalmente o no ${ }^{4}$.

3. Apropiación indebida de las ideas, métodos, resultados o palabras de otra persona, es decir, el uso de la propiedad intelectual de otra persona sin dar los créditos adecuados ${ }^{5}$.

4. Forma de engaño para obtener un crédito inmerecido, como una mejor calificación, por lo que no es ético ni aceptable 6 .

5. Imitar el trabajo de otros con intención deshonesta ${ }^{7}$.

Desde cualquiera de las definiciones que se parta, lo cierto es que el acto de plagio va en contra de un espíritu de confianza e integridad que debe imperar en las actividades académicas ${ }^{8}$ es un tema que concierne a los valores, a la honestidad, esto por cuanto, al realizar un trabajo académico se pueden utilizar diversas fuentes de información, pero se debe respetar y citar adecuadamente cuando las ideas incorporadas en el trabajo no son propias.

El plagio además afecta la calidad, refleja falta de capacidad, de habilidades o talento del estudiante para realizar sus deberes académicos, lo cual se manifestará a futuro cuando se enfrente a diversos retos en el ejercicio profesional.

\footnotetext{
${ }^{2}$ Diccionario de la Real Academia Española, Real Academia Española, acceso: 24 de marzo de 2020, https://dle.rae.es/plagiar\#TIZy4Xb

${ }^{3}$ Greg Ryan et al., «Undergraduate and Postgraduate Pharmacy students, perceptions of plagiarism and academic Honesty», Am J Pharm Educ 73, n. ${ }^{\circ} 6$ (2009): 105, doi: 10.5688/aj7306105

${ }^{4}$ Sheikh Tariq Mahmood, «Intellectual Property Right and Patent: Conceptual Awareness of PhD Students about Plagiarism», conferencia pronunciada en Cairo, Egipto, 2-4 de noviembre de 2010, doi:10.1109/ICEMT.2010.5657562

${ }^{5}$ Lidija Bilic-Zulle et al., «Is There an Effective Approach to Deterring Students from Plagiarizing?», Sci Eng Ethics 14, n. ${ }^{\circ} 1$ (2008): 139-147, doi:10.1007/s11948-007-9037-2

${ }^{6}$ Andrés Carnero et al., «Plagiarism, Cheating and Research Integrity: Case Studies from a Masters Program in Peru», Sci Eng Ethics 23, n. ${ }^{\circ}$ 4(2017):1183-1197. Doi: 10.1007/s11948-016-9820-z

${ }^{7}$ Ibíd.

${ }^{8}$ Deborah Kenny, «Student plagiarism and professional practice», Nurse Education Today 27, n. $^{\circ} 1$ (2007): 14-18. doi: 10.1016/j.nedt.2006.02.004
} 


\section{Formas de plagio}

El plagio se puede cometer de muchas formas, Park $^{9}$ señala una taxonomía de tipos de plagio por parte de los estudiantes que incluye:

1. Realizar trabajos grupales y que un estudiante lo reclame como propio.

2. Enviar trabajos de cursos realizados por un amigo.

3. Copiar el trabajo de otro estudiante y presentarlo como propio (con o sin el conocimiento del estudiante).

4. Copiar el texto sin reconocer su origen.

5. Comprar los trabajos ${ }^{10}$.

Por su parte, Tariq ${ }^{11}$ identifica otros tipos de plagio:

1. Tomar material de internet o de una base de datos electrónicos sin permiso.

2. Adaptar, material con derechos de autor de varias fuentes y mezclarlo para crear un nuevo trabajo sin las citas de la información de origen.

3. No poner comillas, cuando se trata de citas textuales o cambiar la posición de las comillas (debe considerarse las normas de citación).

4. Pretender que una traducción es trabajo propio.

5. Cambiar pocas palabras (sin cambiar la estructura de la oración original).

6. Proporcionar información incompleta respecto a las fuentes.

7. Mezclar información de diferentes fuentes, etc.

Como se observa, la lista no es exhaustiva y debe analizarse a la luz de los casos en concreto.

Se pueden considerar además otras formas que se han ido agregando con la incorporación de los medios tecnológicos, tales como la realización grupal de exámenes en línea (cuando las autoridades esperan que estos sean individualmente desarrollados), suplantación de identidades en la elaboración de pruebas. Adicionalmente se conoce de la compra y venta de actividades académicas, pago por elaboración de pruebas, entre otros.

${ }^{9}$ Ibíd.

${ }^{10}$ Ibíd.

${ }^{11}$ Sheikh Tariq Mahmood. «Intellectual Property...». 
En la práctica, con mucha más dificultad, un estudiante admite el fraude en la evaluación de los aprendizajes cuando este se realiza en beneficio de otros estudiantes, como cuando se le permite a un compañero copiar las respuestas en una prueba, o presentar un trabajo para que otros lo copien.

\section{Factores que inciden en la práctica del plagio}

Algunos factores que incentivan el plagio entre los estudiantes, de conformidad con las investigaciones consideradas, son los siguientes:

1. Estudiantes con pocas habilidades académicas o situaciones de competencia para lograr el éxito ${ }^{12}$.

2. La ignorancia, la oportunidad, la tecnología, la competencia, la falta de reglas y de consecuencias claras ${ }^{13}$.

3. Falta de conciencia sobre la integridad de la investigación y el plagio.

4. Deficiencias generalizadas en las habilidades de escritura y referencia, bajo rendimiento académico y alta tolerancia al plagio ${ }^{14}$.

Es frecuente que se señale que el problema surge porque muchos estudiantes tienen prácticas que no consideran sean deshonestas. Esta afirmación es sustentada en diversas investigaciones:

1. Una de las razones del plagio es el desconocimiento de los estudiantes sobre el concepto de plagio $^{15}$.

2. Muchos estudiantes no sienten que sus prácticas son deshonestas o piensan que sus tutores no podrán detectar esta forma de comportamiento de engaño ${ }^{16}$.

3. Un desafío frecuente es que los culpables no entienden que su comportamiento sea incorrecto ${ }^{17}$.

4. Los estudiantes no percibieron el plagio como un problema grave, como tampoco el uso de estrategias inapropiadas para obtener y reconocer material de otros ${ }^{18}$.

\footnotetext{
${ }^{12}$ Mohamad Awad, Bashar Zogheib y Hamed Alazemi, «A Penalty Scheme for Academic Dishonesty», conferencia pronunciada en Bali, Indonesia, 26-29 agosto de 2013, doi: 10.1109/TALE.2013.6654504

${ }^{13}$ Lidija Bilic-Zulle et al., «Is There an Effective Approach...».

${ }^{14}$ Andrés Carnero et al., «Plagiarism, Cheating...».

${ }^{15}$ Sheikh Tariq Mahmood. «Intellectual Property...».

${ }^{16}$ Deborah Kenny, «Student plagiarism...».

${ }^{17}$ Sheikh Tariq Mahmood. «Intellectual Property...».

${ }^{18}$ Greg Ryan et al., «Undergraduate and Postgraduate...». 
Si bien el plagio existe en todo el orbe en general, respecto a los países en desarrollo se han señalado factores, los cuales fueron recopilados en el trabajo de Carnero et al. ${ }^{19}$, referentes a distintos estudios:

1. Falta de capacitación en la conducta responsable de Investigación (RCR) (Rodríguez y Lolas; Davis; Vasconcelos et al.; Cameron, et al.).

2. Desarrollo deficiente de las habilidades de escritura (Heitman y Litewka; Vasconcelos et al.; Cameron et al.).

3. Tolerancia a la mala conducta durante la educación y las actividades profesionales (Heitman y Litewka; Vasconcelos et al.).

4. Falta de políticas institucionales y supervisión de centros académicos y revistas (Rodríguez y Lolas; Heitman y Litewka; Vasconcelos et al.).

5. Diferentes percepciones de propiedad intelectual y mala conducta (Heitman y Litewka; Davis; Cameron et al.).

6. El efecto generalizado de la corrupción (Heitman y Litewka).

7. Diferencias culturales en los valores (Rodríguez y Lolas; Heitman y Litewka; Davis; Vasconcelos et al.; Cameron et al.).

8. Descuido de las instituciones educativas de la discusión sobre el plagio, integridad académica e investigación, aunado a la falta de desarrollo de habilidades analíticas y de escritura. De conformidad con estos investigadores, estas circunstancias hacen que los estudiantes utilicen el plagio como una estrategia de escritura compensatoria. (Vasconcelos et al.; Heitman y Litewka).

\section{Prevención}

La prevención o anticipación del problema es el tema que más debe ocupar a las autoridades académicas.

El accionar institucional preventivo es muy importante, dado que el plagio no es un tema aislado, va de la mano con los valores, el respeto a los derechos de autor, a referenciar correctamente, aprender a parafrasear (dando crédito de la fuente de la idea, aunque se exprese con palabras propias), citar adecuadamente, incluso cuando el trabajo citado le pertenece al mismo autor, como cuando se presentan trabajos propios ya publicados como si se tratara de una nueva investigación, en cuyo caso se conoce como autoplagio ${ }^{20}$.

La prevención también es importante, ya que existen investigaciones ${ }^{21}$ que indican que si bien las malas prácticas pueden ocurrir en cualquier momento de la carrera de un investigador, con mayor frecuencia ocurren en las primeras etapas (aunque existen pocos estudios respecto a sus orígenes en el pregrado y el posgrado). Para estos autores, las primeras etapas pueden constituir un

${ }^{19}$ Andrés Carnero et al., «Plagiarism, Cheating...».

${ }^{20}$ American Psychological Association, Manual de Publicaciones de la American Psychological Association (Colombia: El Manual Moderno, 2010).

${ }^{21}$ Andrés Carnero et al., «Plagiarism, Cheating...». 
período crítico para prevenir el plagio, cuando los estudiantes comienzan a participar en trabajos de investigación. Es también en estas primeras etapas cuando el plagio puede conllevar un conjunto más amplio de conductas deshonestas como copiar en un examen o tomar los créditos del trabajo de otro.

Por lo señalado es que muchos coinciden en que la formación en ética de la investigación debe integrarse en el plan de estudios de los estudiantes de pregrado y posgrado. De igual manera, las sanciones contra los actos de mala conducta deben hacerse cumplir ${ }^{22}$.

Autores que han estudiado este tipo de faltas académicas han logrado identificar algunas formas de prevención:

a. Compromiso institucional proactivo. La primera medida para prevenir el plagio consiste en el compromiso institucional con la integridad científica y la exteriorización de dicho compromiso a la comunidad estudiantil, es decir, debe existir una actitud proactiva, no simplemente reactiva.

Las instituciones deben tener una política transparente, integral y uniformemente aplicada contra el plagio ${ }^{23}$. Esta política, de «tolerancia cero» contra el plagio incluye la búsqueda de posibles conductas inapropiadas en la investigación, el reporte de los casos sospechosos, la investigación y finalmente la sanción de conformidad con la gravedad del caso ${ }^{24}$.

Para estos autores, el mensaje institucional debe ser claro y fuerte, en el sentido que el plagio es una incorrecta conducta de investigación y, por tanto, no puede ser justificada. Debe concientizarse en las consecuencias adversas del plagio en el largo plazo, como sería el hecho de graduar estudiantes con pocos conocimientos.

Para Culwin ${ }^{25}$, debe existir un proceso de culturización con el fin de que los estudiantes adopten las creencias y valores de los académicos universitarios, se les debe invitar a suscribirse a ellas. Este es el motivo por el que las transgresiones tempranas deben ser respondidas educativamente y no punitivamente.

Por su parte, el personal académico también debe recibir periódicamente capacitación relativa a la mala conducta académica, temas que deben ser discutidos regularmente en las reuniones departamentales ${ }^{26}$.

\footnotetext{
22 Patrick Okonda y Theresa Rossouw, «Prevalence of scientific misconduct among a group of researchers in Nigeria», Developing World Bioethics 13, n. ${ }^{\circ} 13$ (2012): 149-157, doi:10.1111/j.14718847.2012.00339.x

${ }^{23}$ Andrés Carnero et al., «Plagiarism, Cheating...».

${ }^{24}$ Ibíd.

${ }^{25}$ Fintan Culwin, «A Longitudinal Study of Nonoriginal Content in Final-Year Computing Undergraduate Projects», IEEE Transactions on Education 51, n. ${ }^{\circ} 2$ (2008): 189-194, doi: 10.1109/TE.2007.910350

${ }^{26}$ Ibíd.
} 
b. Educación. Otro elemento por considerar es la educación intensiva respecto a la integridad de la investigación y la escritura científica. Esto incluye las sesiones de discusión.

Investigaciones señalan que la educación tiene un impacto mucho más fuerte que las advertencias verbales ${ }^{27}$.

Se debe promover entre los estudiantes los más altos estándares de integridad y ser consientes de cómo evitar un potencial plagio ${ }^{28}$.

Al inicio de los estudios, los estudiantes deberían participar en ejercicios de aprendizaje activo, exhibiciones web y carteles que ilustren el uso apropiado e inapropiado de material no riguroso y sobre el uso de referencias correctas y parafraseos apropiados ${ }^{29}$.

Tariq ${ }^{30}$ propone educar mediante asignaciones para practicar sobre citas a través de diferentes fuentes, enseñar leyes de propiedad intelectual, ofrecer manuales con información sobre las reglas de citar, capacitar a maestros y supervisores de investigación sobre problemas de rastreo y manejo relacionados con el plagio.

La fuerte presión institucional para mantener la integridad del trabajo académico es un determinante crucial de las decisiones de los estudiantes para no plagiar $^{31}$.

c. Establecimiento de códigos de conducta. Estos códigos, deben ser analizados por los estudiantes, profesores e investigadores; por ejemplo, establecer un código de honor, el cual sea firmado por los interesados, para comprometerse con mantener la investigación y la integridad académica, así como informar cualquier caso observado. La ventaja de esta estrategia es la simplicidad de la estrategia y su bajo costo ${ }^{32}$.

El establecimiento de los códigos de honor es una medida promovida y aceptada en diversas investigaciones como una forma para reducir el plagio ${ }^{33}$. Lo gratamente satisfactorio es que los valores inculcados en estas etapas, se llevarán a la vida profesional.

d. Seminarios sobre plagio y escritura científica. Algunos autores señalan que debe haber cursos obligatorios en los centros de estudio, los cuales pueden ser breves, sobre integridad en la investigación ${ }^{34}$. En estos cursos se

\footnotetext{
${ }^{27}$ Lidija Bilic-Zulle et al., «Is There an Effective Approach...».

${ }^{28}$ Ibíd.

${ }^{29}$ Fintan Culwin, «A Longitudinal Study of...».

${ }^{30}$ Sheikh Tariq Mahmood, «Intellectual Property...».

${ }^{31}$ Greg Ryan et al., «Undergraduate and Postgraduate...».

${ }^{32}$ Andrés Carnero et al., «Plagiarism, Cheating...».

${ }^{33}$ Mohamad Awad, Bashar Zogheib y Hamed Alazemi, «A Penalty...».

${ }^{34}$ Andrés Carnero et al., «Plagiarism, Cheating...».
} 
pueden analizar las formas de escritura desadaptativas, como la «redacción de parches», en la que el texto original y prestado se entremezclan ${ }^{35}$, así como la práctica de «copiar / pegar». También es la oportunidad de aconsejar sobre la forma correcta de escribir para que se expresen ideas tomadas de fuentes externas en sus propias palabras, siempre vinculando cada idea con su fuente original $^{36}$.

Los resultados de la investigación de Marxhall, Taylor, Hothersall y Pérez ${ }^{37}$ indican que ni los consejos para evitar el plagio ni las sanciones para los infractores cambiaron la proporción de coincidencia de texto observada en las tareas. Sin embargo, los resultados fueron diferentes luego de un seminario específico de la temática, a partir de ahí se detectó una reducción en las puntuaciones de coincidencia de texto y los casos de plagio.

e. Transformación de las actividades educativas. Esta práctica incluye: desglosar las tareas escritas extensas en tareas múltiples y más pequeñas, para permitir el desarrollo incremental de las habilidades de escritura $^{38}$; utilización de plantillas, para que los estudiantes tengan una guía de elaboración con especificaciones ${ }^{39}$.

Realizar evaluaciones mediante preguntas abiertas ${ }^{40}$ o tipo ensayo ${ }^{41}$.

f. Advertencias. De acuerdo con algunos autores, el plagio puede reducirse a través de las advertencias verbales respecto a sus consecuencias ${ }^{42}$ como podría ser informar a los estudiantes que sus ensayos serían verificados por un software detector de plagio ${ }^{43}$.

Otra vía es la publicación de las sanciones disciplinarias en ${ }^{44}$ el sitio web de las universidades, en sus catálogos académicos ${ }^{45}$.

El uso de la tecnología en las aulas con el fin de prevenir el plagio es otro recurso señalado ${ }^{46}$.

g. Valorar los casos de trampa como oportunidades educativas, en lugar de invertir en estrategias de detección y castigo ${ }^{47}$.

35 Ibíd.

${ }^{36}$ Ibíd.

${ }^{37}$ Tom Marshall et al, «Plagiarism: A case...».

${ }^{38}$ Andrés Carnero et al., «Plagiarism, Cheating...».

${ }^{39}$ Ibíd.

${ }^{40}$ Mohamad Awad, Bashar Zogheib, Hamed Alazemi. «A Penalty...».

${ }^{41}$ Ibíd.

${ }^{42}$ Ibíd.

${ }^{43}$ Lidija Bilic-Zulle et al., «Is There an Effective Approach...».

${ }^{44}$ Mohamad Awad, Bashar Zogheib, Hamed Alazemi, «A Penalty...».

45 Ibíd.

${ }^{46}$ Ibíd.

${ }^{47}$ Greg Ryan et al., «Undergraduate and Postgraduate...». 
Si bien se pueden asumir diferentes medidas de prevención para el plagio, algunas investigaciones ${ }^{48}$ concluyen que la más importante de todas es promover la integridad académica y la honestidad, esto incluso antes de sufrir las consecuencias por el plagio.

En la UNED, después de haber dado inicio a un proceso disciplinario contra una estudiante, la cual aceptó los hechos, pero manifestó desconocer que brindar las respuestas de su examen a los compañeros constituía un plagio académico, se le motivó a aceptar una sanción alterna y en este caso consistió en la elaboración de un video con la colaboración de la Defensoría de los Estudiantes para alertar a los estudiantes que dicha práctica no estaba permitida y era sancionable.

Enseñar a los estudiantes cómo evitar el plagio aplicando reglas claras, el código de ética y la conciencia de responsabilidad pueden contribuir en mayor medida a la reducción de la mala conducta académica.

\section{Detención y sanción}

Como ha quedado claro del apartado anterior, lo primordial es trabajar en la etapa de la prevención. Sin embargo, a continuación se hace una breve mención a la detención y sanción del plagio.

Algunos tipos de plagio pueden ser prevenidos mediante la tecnología de la información, la cual proporciona recursos que facilitan la detención e investigación de plagio, una vez que las tareas o investigaciones han sido presentadas para su evaluación.

Para considerar que existe plagio, Bilic-Zulle ${ }^{49}$ expresan que se debe considerar plagio cuando el documento del estudiante contiene más de un $12.5 \%$ de copia textual de acuerdo al software utilizado para detectarlo. Es importante señalar que el software es un recurso que brinda un porcentaje como resultado de copia, el cual luego hay que validar «a pie» para constatar que se han indicado las citas correspondientes.

En esta etapa, también debe considerarse la existencia de una protección adecuada para las personas denunciantes, esto alentará la notificación de casos sospechosos de mala conducta sin temor de una reacción violenta contra la persona accionante ${ }^{50}$.

El plagio debe tener consecuencias, indistintamente de las formas en que este pueda presentarse. Cada institución educativa, mediante el reglamento interno, debe establecer las sanciones para las prácticas deshonestas, en el caso del Reglamento General Estudiantil de la UNED, existe además la

${ }^{48}$ Lidija Bilic-Zulle et al., «Is There an Effective Approach...».

${ }^{49}$ Ibíd.

${ }^{50}$ Patrick Okonda, Theresa Rossouw, «Prevalence of scientific...». 
posibilidad de medidas alternas cuando el estudiante acepta la responsabilidad durante el proceso.

De parte de los estudiantes, no cabe alegar ignorancia de la normativa o solicitar la consideración de si hubo desconocimiento o intencionalidad en los hechos, de ahí la importancia de advertir respecto a esta materia a los jóvenes que inician sus estudios académicos.

El problema del plagio se complica si existe tolerancia a este. Esta tolerancia puede manifestarse como la falta de sanciones o la disminución de las sanciones a simples reprimendas, argumentando que copiar sin indicar la fuente es un comportamiento natural de los estudiantes o que la enseñanza consiste en una repetición constante de ideas externas ${ }^{51}$.

Estos autores resaltan la necesidad de combinar la educación intensiva contra el plagio con políticas de respuesta más fuertes, sin embargo y en este punto del discurso, nuevamente volcamos la mirada hacia la prevención, ya que la educación y apropiación de valores por parte de los estudiantes, constituirán un patrimonio que atesorarán a lo largo de la vida profesional.

\section{Caso Costa Rica: Resolución de interés}

El 6 de noviembre del 2020, mediante resolución, 21429-2020, la Sala Constitucional resolvió un recurso de amparo relativo a la viabilidad o no de las actividades virtuales universitarias. Esta resolución es importante y será un buen instrumento para prevenir el fraude académico, porque respalda a las universidades para elaborar lineamientos que facilitan los controles. En esta resolución nuevamente se realiza un reconocimiento a la Autonomía Universitaria en lo referente a la regulación de las evaluaciones académicas, al respecto indica:

conviene señalar que no corresponde a este Tribunal determinar como los estudiantes universitarios deben ser evaluados, pues ello es competencia del centro educativo y de las personas docentes. Es decir, no procede analizar si las evaluaciones deben ser efectuadas de forma sincrónica o asincrónica, como se indicó es competencia del centro académico (sic) (la negrita no es del original).

El caso es relevante, por cuanto a raíz de la pandemia y el hecho que las universidades han tenido que implementar evaluaciones virtuales, los fraudes por copia en los exámenes han proliferado, habiéndose constatado casos en los que se realizan grupos de WhatsApp masivos y se comparten las

\footnotetext{
${ }^{51}$ Andrés Carnero et al., «Plagiarism, Cheating...».
} 
respuestas a las pruebas, desvirtuando los objetivos de la prueba. Esto puede ocurrir principalmente en exámenes asincrónicos, o bien, los sincrónicos donde no se obligue a mantener la cámara y el audio encendidos.

Uno de los aspectos relevantes del análisis es el planteamiento de la prevalencia o no del derecho de la imagen y a la intimidad sobre el derecho a la educación superior a través de tecnologías de la información. La resolución de la Sala es insuficiente, pues se debe analizar cada caso en concreto, pero sí se trazan coordenadas que permiten a los centros educativos analizar las diversas situaciones que se presenten en el marco general de la autonomía universitaria.

Algunos aportes importantes son:

1. Las universidades tienen el respaldo constitucional de la Autonomía Universitaria para determinar las formas de evaluación.

2. Las universidades pueden regular sus lineamientos académicos y administrativos para la docencia con componente virtual, siempre y cuando se tome en cuenta el derecho a la intimidad, privacidad y respeto de los derechos fundamentales de las personas estudiantes.

3. Es viable la normativa que dicte la obligatoriedad de actividades académicas por realizarse de forma virtual, sincrónica o asincrónica. La inasistencia de los estudiantes queda sujeta a la respectiva justificación según el reglamento.

4. Es posible la obligatoriedad de habilitar el video y el audio, con el fin de verificar que la identidad de la persona que está realizando la prueba coincida con la información de la persona estudiante matriculada. En el caso en particular, los lineamientos de la UCR, indican: «El video y audio deberá habilitarse como garantía de la correcta y ética realización de la prueba. La persona docente o la Unidad Académica debe garantizar que la persona estudiante cuente con las condiciones de acceso tecnológico para realizar la prueba».

5. No es viable que el estudiante alegue imposibilidad de cumplir con una prueba sincrónica con video y audio, justificando lo anterior con el hecho que se ve afectado anímica o psicológicamente ya que:

«(...) no es de recibo el argumento cuando afirma que "no me siento bien ni cómodo mostrando mi imagen'. Al respecto, la Sala indica que de no existir la situación excepcional que se atraviesa e impone el distanciamiento físico, 'el recurrente tendría que presentarse a rendir la prueba junto con el resto de compañeros y compañeras e impedirían que se mantuviera como incógnito'». 
6. No es de recibo alegar que está siendo objeto de injerencias arbitrarias o abusivas en su vida privada, la de su familia o domicilio, que se ve amenazada su dignidad como persona cuando debe realizar pruebas sincrónicas, o que se viola su derecho de imagen y debido proceso. Lo anterior, ya que son situaciones que se pueden resolver: acudiendo a lugares públicos o privados a realizar la prueba, poner un fondo ficticio en la plataforma que utiliza, estableciendo medios para que en caso de grabarse la imagen y la voz, se prevea la forma de resguardar las mismas.

En otras palabras, no hay ningún roce con la Constitución cuando la universidad establece pruebas virtuales asincrónicas o sincrónicas. Las pruebas que pudieran presentar problemas para su realización y por las cuales se debe analizar el caso en concreto, son aquellas sincrónicas cuando se graba la imagen y la voz. Pero en estos casos, el estudiante debe justificar las razones por las cuales, su situación personal le impida ingresar con audio o video a la sesión, el caso debe analizarse en concreto, considerando diversas formas de resolver el inconveniente, pero tampoco existe impedimento en términos generales, si se ha previsto la forma de resguardar las grabaciones. Es decir, no se puede argumentar en abstracto debiendo cada estudiante exponer y probar situaciones concretas que le estén afectando, cuando se trata de pruebas en las cuales su imagen o su voz puedan ser grabadas.

El tema se encuentra en pleno desarrollo, pero esta resolución es el punto de inicio para el análisis casuístico que las universidades en Costa Rica deben desarrollar a lo interno. Es un buen instrumento de apoyo con el fin de buscar nuevas formas de contrarrestar los fraudes, lo importante es el apoyo que la Sala da a la Autonomía Universitaria.

A futuro, conforme se presenten los casos, tal y como lo señaló la defensa de la UCR, deberá considerarse la multiplicidad de facetas a que está sujeta la docencia en el ámbito universitario, iniciando por «la diversidad disciplinar, la libertad de cátedra, la libertad de pensamiento y opinión, la complejidad de la realidad, la flexibilidad curricular (...)».

\section{Conclusiones}

El tema del plagio, en sus múltiples formas, cobra importancia gracias a las facilidades tecnológicas de hoy.

La circunstancia actual de la pandemia obligó a la virtualización masiva de las asignaturas, en un plazo muy corto, lo que llevó a la intensificación del uso del internet y facilitó las malas prácticas de los estudiantes como la copia en los exámenes en línea, suplantación de identidades en la elaboración de pruebas, copia desmesurada de trabajos, entre otros. 
La situación es compleja, desenmascara muchos problemas conexos como: la pérdida de valores, como la honestidad, la falta de habilidades académicas por parte de los estudiantes, la ambigüedad existente, ya que existen prácticas que los propios estudiantes no consideran como deshonestas, muchas veces por desconocimiento, o bien, porque desde las propias instituciones educativas no se le ha dado la importancia debida al aprendizaje y uso adecuado de las estrategias para obtener y reconocer el material ajeno o ha habido tolerancia ante los usos inadecuados o prácticas indebidas.

En este texto se ha resaltado la importancia del trabajo preventivo por parte de las instituciones académicas, primordialmente en las primeras etapas universitarias. Las investigaciones consultadas refieren a la importancia de la proactividad por encima de la reactividad.

Las recomendaciones van dirigidas al establecimiento de políticas de cero tolerancia, algunos hablan de un proceso de culturalización para que el estudiante adopte creencias y valores de un universitario. El personal académico también debe ser capacitado y las actividades educativas deben repensarse, replantearse y transformarse de acuerdo a las circunstancias actuales.

Al estudiantado debe guiársele para que adopte una actitud íntegra en su accionar, en la investigación y en la escritura académica. Deben participar de un aprendizaje activo y debe existir una fuerte presión institucional para mantener la integridad en los trabajos académicos, tales como el establecimiento de códigos de conducta, los cuales, como lo señalan los autores, son procedimientos sencillos y de bajo costo. Otra forma es mediante cursos o seminarios sobre plagio y escritura académica, que conlleva aprender del respeto a los derechos de autor, referenciar correctamente, parafrasear citando adecuadamente, entre otros.

Mucho más efectividad ha demostrado el trabajo preventivo que las advertencias verbales, la detención y sanción (que además son tratamientos individuales del problema) mientras que la prevención se trabaja de manera generalizada.

La prevención además de ser más económica que una investigación y el desarrollo de procesos disciplinarios, no atrae daños colaterales como pueden darse hacia las personas denunciantes, pero y ante todo, la educación y apropiación de valores serán patrimonio para los estudiantes que les guiarán a lo largo de la vida profesional. 
La detención y sanción son etapas que conllevan mucho desgaste entre los actores y si se llega a estas instancias no se puede permitir justificar el accionar debido a la ignorancia de la normativa, el desconocimiento o falta de intencionalidad.

Otro aspecto por resaltar es que cuando el plagio se detecta, no debe descartarse transformar el proceso en una oportunidad educativa. En todo momento, la institución debe actuar como formadora, insistiendo en la ética de la investigación, con un compromiso proactivo a favor de la honestidad.

Por otro lado, es de relevancia la reciente resolución del 6 de noviembre del 2020 de la Sala Constitucional por cuanto, de forma clara, plantea que las universidades, dado el Principio de Autonomía Universitaria, pueden normar $\mathrm{y}$ establecer las condiciones en las cuales realizan pruebas virtuales asincrónicas o sincrónicas, debiendo analizarse cada caso en concreto, cuando se alegue que se ve perjudicado su derecho a la imagen, pero sin inconveniente cuando la universidad prevé la forma de resguardar la imagen y la voz del estudiante. Sin duda, esta resolución aporta de forma importante en las decisiones que las instituciones educativas deben tomar con el fin de prevenir el fraude académico.

El estudio realizado espera contribuir mediante alternativas viables que pueden realizarse para prevenir el plagio y debe inquietar respecto a cuánto trabajo falta ejecutar en las instituciones con el fin de desarrollar, en los estudiantes, la concientización de lo importante que es el conocimiento y la demostración y comprensión de lo aprendido para un desarrollo profesional pleno.

Este estudio bibliográfico, de carácter descriptivo y exploratorio, deja planteados muchos temas para el desarrollo. En el plano preventivo y en la normativa y lineamientos universitarios que deben ser revisados para sustentar las nuevas circunstancias que se plantean, especialmente por la experiencia que ya se empieza a generar a partir de la generalización de las pruebas virtuales. 


\section{Formato de citación según APA}

Vargas-Morúa, E. (2021). El plagio: consideraciones para su prevención. Revista Espiga, 21 (41), páginas 68-85.

\section{Formato de citación según Chicago-Deusto}

Vargas-Morúa, Elizarda. «El plagio: consideraciones para su prevención». Revista Espiga 21, n. ${ }^{\circ} 41$ (enero-junio, 2021): páginas 68-85.

\section{Referencias bibliográficas}

American Psychological Association. Manual de Publicaciones de la American Psychological Association. Colombia: El Manual Moderno, 2010.

Awad, Mohamad, Bashar Zogheib y Hamed Alazemi. «A Penalty Scheme for Academic Dishonesty». Conferencia pronunciada en Bali, Indonesia, 26-29 de agosto de 2013. doi: 10.1109/TALE.2013.6654504

Bilic-Zulle, Lidija, Josip Azman, Vedran Frkovic y Mladen Petrovecki. «Is There an Effective Approach to Deterring Students from Plagiarizing?», Sci Eng Ethics 14, n. ${ }^{\circ} 1$ (2008): 139-147. doi: 10.1007/s11948-007-9037-2

Carnero, Andrés; Percy Mayta-Tristan, Konda Kelika, Edward Mezones-Holguin, Antonio Bernabe- Ortiz, German Alvarado, Carlos Canelo Aybar, Jorge Maguiña, Eddy Segura, Antonio Quispe, Edward Smith, Angela Bayer, Andrés Lescano. «Plagiarism, Cheating and Research Integrity: Case Studies from a Masters Program in Peru», Sci Eng Ethics 23, n. ${ }^{\circ} 4$ (2017):1183-1197. Doi: $10.1007 / \mathrm{s} 11948-016-9820-\mathrm{z}$

Culwin, Fintan. «A Longitudinal Study of Nonoriginal Content in Final-Year Computing Undergraduate Projects». IEEE Transactions on Education 51, n. ${ }^{\circ}$ (2008): 189-194. doi: 10.1109/TE.2007.910350

Kenny, Deborah. «Student plagiarism and professional practice». Nurse Education Today 27, n. ${ }^{\circ} 1$ (2007): 14-18. doi: 10.1016/j.nedt.2006.02.004

Mahmood, Sheikh Tariq. «Intellectual Property Right and Patent: Conceptual Awareness of $\mathrm{PhD}$ Students about Plagiarism». Conferencia pronunciada en Cairo, Egipto, 2-4 de noviembre de 2010. doi: 10.1109/ICEMT.2010.5657562 
Marshall, Tom, Beck Taylor, Ellie Hothersall, Leticia Pérez-Martín. «Plagiarism: A case study of quality improvement in a taught postgraduate programme». Medical Teacher 33, n. ${ }^{\circ} 7$ (2011): 375-381. doi:

10.3109/0142159X.2011.579201

Okonda, Patrick, Theresa Rossouw. «Prevalence of scientific misconduct among a group of researchers in Nigeria». Developing World Bioethics 13, n. ${ }^{\circ} 13$ (2012): 149-157. doi:10.1111/j.1471-8847.2012.00339.x

Price, Bob. «Avoiding plagiarism: guidance for nursing students». Europe PMC 28, n. ${ }^{\circ} 26$ (2013): 45-51. doi: 10.7748/ns2014.02.28.26.45.e8514

Real Academia Española. Diccionario de la Real Academia Española. Rae.es. Acceso: 24 de marzo de 2021. https://dle.rae.es/plagiar\#TIZy4Xb

Ryan, Greg; Helen Bonanno, Inés Krass, Karen Scouller, Lorraine Smith. «Undergraduate and Postgraduate Pharmacy students, perceptions of plagiarism and academic Honesty». Am J Pharm Educ 73, n. ${ }^{\circ}$ (2009): 105. doi: 10.5688/aj7306105

Sala Constitucional de la Corte Suprema de Justicia de Costa Rica. STC 214292020 del 6 de noviembre de 2020. 